Investieren statt spenden - neue Unterstützungsformen in der Diakonie

Marc Baumann

\section{Investieren statt spenden - neue Unterstützungsformen in der Diakonie}

Spenden und investieren folgen verschiedenen Grundgedanken. Während Spenden Wohltätigkeitsakte sind und nicht zurückbezahlt werden müssen, erfolgen Investitionen marktbasiert und unterliegen einer Rückzahlungspflicht. Auf den ersten Blick scheinen diese Welten unvereinbar. Bei genauerer Betrachtung ergeben sich jedoch interessante Synergien. ${ }^{1}$

\section{Spenden und investieren}

Die Spende ist ein Akt klassischer Wohltätigkeit und zeichnet sich dadurch aus, dass eine wohlhabende Person einer bedürftigen Person hilft. Damit verbunden ist ein Gefälle zwischen Spender und Empfänger. Der Empfänger unterliegt keiner Rückzahlungs-, jedoch meist implizit einer Dankbarkeitspflicht. Die Investition schafft ein anderes Beziehungsgefüge. Sie erfolgt marktbasiert und muss zurückbezahlt werden. Der Investor investiert in ein Projekt, weil er dem Kapitalnehmer vertraut, dass dieser mit den erhaltenen Mitteln einen Ertrag erwirtschaftet und das Kapital wieder

1 Dieser Beitrag erschien zuerst auf dem Fachportal der Konferenz Diakonie Schweiz, URL: https://www.diakonie.ch/investieren-statt-spenden-neue-unterstuetzungsformen-in-der-diakonie/ (abgerufen am 22.04.2021).

Jahrbuch Diakonie Schweiz 4 (2020/2021) - ISSN 2504-3994

Dieser Text ist lizenziert unter einer Creative Commons Namensnennung 4.0 International Lizenz (CC BY 4.0): (https://creativecommons.org/licenses/by/4.0/). zurückbezahlt. Die Investition begründet eine länger dauernde und partnerschaftliche Beziehung. Die Rückzahlungspflicht und die Knappheit der Mittel führen zu stärkerer Verantwortung und Kooperation. Spenden und investieren sind dennoch keine Gegenspieler, sondern haben je ihre Einsatzfelder. Ein gutes Beispiel dafür sind die Aktivitäten der Christlichen Ostmission in Rumänien.

Die Christliche Ostmission ist seit Jahrzehnten in Rumänien tätig. In der Zeit bis zum Sturz von Nicolae Ceausescu im Jahr 1989 konzentrierten sich die Tätigkeiten auf den Transport von Hilfsgütern, Bibeln und die Unterstützung von Kinderheimen. Die Öffnung des Landes im Jahr 1989 führte zu neuen Freiheiten, die für die im Kommunismus gross gewordenen Menschen eine Herausforderung darstellten. Es fehlten die Erfahrung und das Wissen für den Aufbau von wirtschaftlichen Strukturen sowie für die Gründung und die Organisation von Unternehmen. Gleichzeitig wollten viele Menschen schnell zu materiellem Wohlstand gelangen. Diese Situation führte dazu, dass viele Fördergelder nicht für einen nachhaltigen Wirtschaftsaufbau, sondern für den Erwerb von Konsumgütern verwendet wurden.

In dieser Situation war es entscheidend, dass die Fördergelder als Investitionen ausgestaltet wurden. Die zweckkonforme Verwendung und die Rückzahlung der Gelder wurden durch Pfandrechte, durch eine enge Begleitung der Kapitalnehmer und durch Pflichtausbildungen in Unternehmensführung und Geschäftsethik sichergestellt. Der Erfolg war derart durchschlagend, dass der Organisation auch öffentliche Gelder anvertraut wurden, die bisher mit einer Spendenlogik annähernd wirkungslos verpufften.

Der Geldsegen führte dazu, dass man die Mikrofinanzbank Romcom SA gründete. Die Bank, die mehrheitlich einer gemeinnützigen Stiftung in Rumänien gehört, wurde in der Folge unter Mithilfe der auf ethische Vermögensanlagen spezialisierten Vermögensverwalterin Invethos AG mit Sitz in Bern weiter ausgebaut. Die Romcom SA ist ein gutes Beispiel für einen Übergang von spendenbasierten Programmen zu einer Investitions- 
strategie. Letztendlich verfolgen beide dieselben Ziele. Sie wollen Menschen am Rande der Gesellschaft eine würdige Existenz und Teilhabe am sozialen Leben ermöglichen. Es gibt jedoch eine Zeit des Spendens und eine Zeit des Investierens.

\section{Soziale Investitionen}

Investition in Firmen wie die Romcom SA bezeichnet man als soziale Investitionen oder als Impact Investments. Solche Investitionen kombinieren die Erreichung von sozialen Zielen mit einer markbasierten Investitionslogik. Sie zeichnen sich dadurch aus, dass die sozialen Ziele durch aktive Massnahmen erreicht werden sollen. Sie grenzen sich deshalb vom grossen Meer der nachhaltigen Anlagen ab, die eher nach dem Prinzip der Schadensvermeidung funktionieren. Sie sind von der Überzeugung getragen, dass Überlegungen aus der Investitionswelt helfen können, knappe Mittel bestmöglich und resultatorientiert einzusetzen. Die Ausgestaltung als Investition eröffnet zudem die Chance, dass an der Lösung sozialer Probleme alle Beteiligten mitarbeiten. Ein Beispiel dafür ist der Social Impact Bond im Kanton Bern, den die Unternehmervereinigung Fokus Bern zusammen mit der Invethos AG im Jahr 2015 aufgelegt hat.

\section{Social Impact Bond des Kantons Bern}

Der Social Impact Bond des Kantons Bern (SIB) hat zum Ziel, anerkannte Flüchtlinge und vorläufig Aufgenommene in den Arbeitsmarkt zu integrieren und diese auszubilden. Die Messung der Erfolgsquote erfolgt gegen vordefinierte Integrations- und Ausbildungsziele. Werden diese erreicht oder übertroffen, so erhalten die Investoren ihr Kapital und eine Verzinsung ausbezahlt. Werden die Integrationsziele nicht erreicht, so verlieren die Investoren einen Teil ihres Kapitals und erhalten keine Verzinsung. Dasselbe gilt für den Leistungsbringer, für den ebenfalls eine Bonus- und Maluskomponente gilt. Damit soll auf Seiten des Leistungserbringers ein
Anreiz für die Erreichung der Erfolgsquote gesetzt und umgekehrt der Anreiz, Klienten länger in einer Institution zu halten, vermindert werden.

Ein weiteres Ziel des SIB ist, verschiedene Methoden der Integration miteinander zu vergleichen. SIBs wirken idealerweise innovationsfördernd und regen zur Experimentierfreude an. Zu Letzterem eignet sich der SIB auch deshalb gut, weil bei einem Misserfolg die Kosten der öffentlichen Hand sinken und die Verluste für einmal bei den Investoren privatisiert werden. Der SIB entfaltet seine Wirkung dann am besten, wenn neue Lösungsansätze den Miteinbezug des privaten Sektors erfordern. Der SIB gleicht in seiner Ausgestaltung einer Public Private Partnership. Im SIB des Kantons Bern äussert sich das durch den Miteinbezug der Unternehmervereinigung Fokus Bern, die als Bindeglied zur Wirtschaft bei der Stellensuche mithilft. Der SIB ist auch ein Bekenntnis dazu, dass gewisse Herausforderungen nur gemeinsam gelöst werden können und den Beitrag aller erfordern.

\section{Diakonie}

Vielleicht können die Gedanken zum Thema spenden und investieren einen Anstoss geben, im Bereich der Diakonie über neue Unterstützungsformen nachzudenken und experimentierfreudiger zu werden. Der Miteinbezug des privaten Sektors kann der Problemlösung dienen und Mittel freisetzen, die die Wirkungsreichweite erheblich vergrössern. Die Denkweise des Investierens ist gekennzeichnet durch das grosse Vertrauen, das man seinem Gegenüber entgegenbringt. Dieses Gegenüber ist ein Partner auf Augenhöhe, dem man selbständiges und verantwortliches Handeln zutraut. Darin liegt eine enorme Aufwertung der Person. Ziel jedes sozialen Investierens ist die Freisetzung und Selbständigkeit des anderen und nicht dessen Abhängigkeit. Daran sollte sich auch diakonisches Handeln messen.

Autor:

Marc Baumann, Rechtsanwalt, Notar, $M B A, E M B A, C E O$ Invethos $A G$ 